\title{
Deep Laser Cooling and Trapping of Sr at VNIIFTRI
}

\author{
K. Khabarova ${ }^{1,3,4}$, S. Strelkin ${ }^{1,2}$, A. Galyshev ${ }^{1,2}$, O. Berdasov ${ }^{1,2}$, A.Gribov ${ }^{1,2}$, N. Kolachevsky ${ }^{1,2,3}$, \\ and S. Sluysarev ${ }^{1,2}$ \\ ${ }^{1}$ VSUE VNIIFTRI, Mendeleevo, Moscow Region, Russia \\ ${ }^{2}$ National Research Nuclear University MEPhl, Moscow, Kashirskoye sh. 31, Russia \\ ${ }^{3}$ P.N. Lebedev Physical Institute, Moscow, Leninsky prospekt 53, Russia \\ ${ }^{4}$ Russian Quantum Center, Moscow region, Skolkovo, Novaya st. 100A, Russia
}

Today strontium optical lattice clock is one of the most stable source of frequencies approaching eighteenth digit in fractional stability [1]. Such impressive performance makes strontium optical clock a strong candidate for re-definition of second and open new perspectives for fundamental tests and applications. Our group at VNIIFTRI works on development of Sr-87 lattice clock targeting fractional inaccuracy of frequency at $10^{-16}$ level.

In this talk the current progress of past few years will be discussed: efficient first stage laser cooling at $461 \mathrm{~nm}$, second stage laser cooling at $689 \mathrm{~nm}$, development of stabilized lasers for the second stage cooling and clock transition spectroscopy, trapping atoms into the dipole trap. Using highly stabilized laser at $689 \mathrm{~nm}$ [2] we demonstrated deep second stage laser cooling at the "broadband" and "single mode" regimes for Sr-88 isotope. Achieved temperatures of 2-3 $\mu \mathrm{K}$ are sufficient for efficient loading into an optical dipole trap at the magic wavelength of $813 \mathrm{~nm}$ (see fig. 1). After test experiments with Sr-88 we plan to switch to less abundant $\mathrm{Sr}-87$ isotope for spectroscopy of the clock transition at $698 \mathrm{~nm}$.

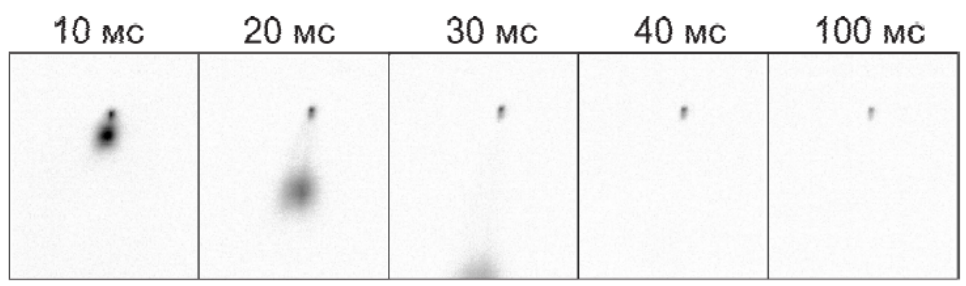

Figure. 1: Retrapping from the MOT to the optical dipole trap of Sr-88 cloud. Images are captured after 10, 20, 30, 40 and $100 \mathrm{~ms}$ after switching off the MOT.

\section{References}

1. B.J. Bloom et al., Nature 506, 71 (2014)

2. K. Khabarova et al., Quantum Electronics 42, 1021 (2012)

This is an Open Access article distributed under the terms of the Creative Commons Attribution License 4.0, which permits unrestricted use, distribution, and reproduction in any medium, provided the original work is properly cited. 